\title{
The Diviner and the Scientist: Revisiting the Question of Alternative Standards of Rationality*
}

\author{
BRIAN EPSTEIN
}

Journal of the American Academy of Religion 78, No. 4 (2010), 1048-1086.

Are the standards of reasoning and rationality in divination, religious practice, and textual exegesis different from those in the sciences? Can there be different standards of reasoning and rationality at all? The intense "rationality debate" of the 1960s, 70s, and 80s focused on these questions and the related problems of relativism across cultures and systems of practice. Although philosophers were at the center of these debates at the time, they may appear to have abandoned the question in recent years. On the contrary, I discuss recent developments in philosophy that approach the issue from a number of new directions, changing our understanding of reasoning and rationality. I argue that, in comparing the diviner to the scientist, focusing on reasoning is likely to be a red herring. Instead, I argue that a careful treatment of rationality, paying particular attention to its context-dependence, untangles longstanding confusions. Moreover, it points the way forward to investigating modest but interesting ways for there to be alternative standards of rationality.

When a Zande boy in his household was bitten on the foot by a snake, the anthropologist E.E. Evans-Pritchard reports, a healer was rushed over who "knew exactly what was required." The healer took out a knife, a piece of bark, and some grass. He chewed some of the bark and had the boy chew the rest, swallowing the juice and spitting out the wood. Then they did the same with the grass. Finally, the healer made incisions on the boy's foot, and

* I am grateful to Jonathan Schofer, Robert Sugden, Stephen White, Ronald Parr, Mark Richard, Bill Fitzpatrick, Jody Azzouni, and two anonymous referees for their comments. 
sucked at them. Before long, the healer began to belch, and announced the boy would recover (1934: 54-55).

Evans-Pritchard asked the healer why he had chewed some bark and grass himself, rather than just administering them to the boy. The healer gave two reasons. First, as insurance. In case the boy had died, he wanted to avoid being accused of having given the boy bad medicine. Second, because he had spoken to the piece of bark in advance. He had asked the bark to make him belch if the boy would recover, and to make him refrain from belching if the boy would not. And according to the healer, the bark did its job, correctly foretelling the boy's recovery.

With this example Evans-Pritchard highlights the complex relations between "mystical” and natural causation in Zande explanations of events. For certain phenomena, the Azande carefully separate the two, while for other phenomena they treat mystical and natural causes on a par. The case also gives Evans-Pritchard material for explicating elements of Zande reasoning. In developing therapies, he argues, the Azande explicitly perform experiments and assess their results on empirical grounds.

The healer's procedure, it seems, is easy to explain from a strictly scientific perspective. The procedure involves multiple steps, and chances are that as a whole it is reasonably effective. It is possible that the only medically relevant step is sucking the poison out of the wound. While the other elements are hypothesized to be effective, they may not be. Or it may be that eating the bark and grass does have medicinal properties. Having spoken to the bark may be seen as a similar hypothesis. If current scientific knowledge is remotely correct, it is untrue that speaking to the bark will have medicinal consequences. But the fact that the diviner does so can easily be interpreted as his acting on just another, albeit false, scientific hypothesis. This “intellectualist" interpretation of magical or divinatory practices as proto-science has its roots at least as far back as Tylor (1871).

Evans-Pritchard himself rejects this interpretation, insisting that one should not collapse Zande magic into Zande science. However, he also rejects Levy-Bruhl's attempt to mark a sharp distinction between the 
scientific reasoning system of moderns and the "prelogical" reasoning systems of "primitive" cultures. Retaining the dichotomy between magical and naturalistic thinking, he comes to the unsatisfying conclusion that Zande medicine involves both sorts of reasoning, sometimes operating redundantly, sometimes complementing one another, and sometimes consisting exclusively of one or the other.

The interpretation of such phenomena as Zande divination was at the center of a remarkable conversation among philosophers, anthropologists, religion scholars, political scientists, psychologists, linguists, and literary theorists from the 1950s through the early 80s. The well-known "rationality debates" centered on the nature of reasoning, the possibility of different world-views, and the respective roles of reason, religion, magic, and science in belief and action. In 1982, Martin Hollis and Steven Lukes presented the essays in their collection Rationality and Relativism as a snapshot taken in the midst of a dynamic debate, "a report from part of a lively and changing front." (1982: 2) The anthropologist Robert Ulin expressed similar sentiments in Understanding Cultures of 1984, presenting the AngloAmerican rationality debates set against work in hermeneutics and critical theory.

But in retrospect, these works seem to mark an ending rather than a flourishing. By the mid-80s the debate was already reaching stasis, with attention shifting away from rationality, among those still concerned with relativism. ${ }^{1}$ Ulin comments in the recent edition of Understanding Cultures that these questions "no longer command the degree of attention that they did in the 1970s and 1980s." (2001: 1)

There are many reasons for this. In some disciplines, suspicions arose about the project of one culture interpreting another from a detached standpoint. This was accompanied by turns to reflexivity, and worries about

${ }^{1}$ Other collections at the time addressing relativism, such as Meiland and Krausz (1982), address similar issues to those discussed in Hollis and Lukes (1982), while later collections, such as Krausz and Meiland (1989) and Krausz and Shusterman (1999), scarcely mention reason, rationality, or epistemic norms. 
the centrality of "culture" in social theory altogether. The views of AngloAmerican philosophers on the topic were changed for rather different reasons. For the better part of the $20^{\text {th }}$ century, professional philosophers had largely been driving the debates on rationality and culture. ${ }^{2}$ Both philosophical innovations and empirical work in linguistics, psychology, and cognitive science, however, seem to have led philosophers away from the issues altogether. $^{3}$

Yet contrary to appearances, these developments have led philosophers not to disengage from the study of rationality, but to change the way the debates have taken place. The rationality debates, from the perspective of contemporary philosophy, seem antiquated. One of the central confusions that permeated the rationality debates - and that we now have the resources to avoid - is the failure to distinguish reasoning from rationality. To reason is to draw inferences, to move from a set of beliefs or claims to another set of beliefs or claims. Rationality is a broader notion, indicating a kind of

${ }^{2}$ From Levy-Bruhl and Popper to Wittgenstein to Quine, Davidson, Winch, MacIntyre, Taylor, Rorty, Hacking, Jarvie, Elster, Hollis, and others.

3 A number of factors are commonly seen as reasons for the decline of the rationality debates in philosophy. One is the resounding impact of Donald Davidson's paper, “On the Very Idea of a Conceptual Scheme” (1973). Equally important the series of negative empirical results in linguistics and cognitive psychology, refuting the exciting claims made by Whorf in the 40s. Work in linguistics has largely disposed of Whorf's claims, and work in cognitive and experimental psychology has also shattered hopes of finding radical differences of the sort people had once looked for, such as in color categorization. Schlesinger 1991 discusses some of this history. Some recent work (e.g., Lucy 1996, Boroditsky 2001) provides some promising evidence for the link between language and thought differences, but even if correct, the claims are much more modest than had been widely expected and believed in mid-century. A third critical reason is ongoing challenges to Kuhn's (1962) claims about "incommensurable paradigms" in science, particularly those posed by the birth and widespread adoption of externalism in the philosophy of language and the philosophy of mind. Kuhn himself took the challenges posed by Putnam 1975 very seriously, and spent a good part of the next decades addressing them. See Kuhn, Conant, and Haugeland 2000. 
rightness or faultlessness in forming a belief or choosing an action. To reason well may be one way of forming rational beliefs or guiding action, but it is not the only way. It may, for instance, be rational to form a belief based on one's sense perceptions, or based on the testimony of others, no reasoning involved. Thus the two questions, does the diviner reason differently from the scientist? and does the diviner have alternative standards of rationality from the scientist? may have entirely different answers.

Because so much attention has been paid to reasoning, I begin with an examination of some of the difficulties even in identifying the reasoning involved in the diviner's practice. Casting the problem as a difference between forms of reasoning raises a thicket of problems. And in the end, it is likely to tell us little about the issues that have traditionally concerned theorists of cultural difference. Moving beyond reasoning, I turn to exploring ways that recent work on rationality may inform the question of whether what I will call "rationality pluralism" - i.e., the existence of alternative standards of rationality - is possible.

I briefly examine practical and theoretical rationality, focusing in particular on the variety of ways rationality has recently come to be understood as context-dependent. Among several sorts of contextdependence, I discuss ways the norms of rationality plausibly depend on cultural factors. Subsequently, I criticize the widely-used taxonomy of views of rationality given by Hollis and Lukes in Rationality and Relativism, and present a different and more promising approach to the possibility of alternative standards of rationality.

This topic - the context-dependence of the norms of rationality - is an abstract one. Treated in the general way it is addressed in the philosophical literature, its application to the cross-cultural study of religion and ethnography can be opaque. Diving into the role of context in rationality in general lacks the vividness and excitement of a rich ethnographic study, such as Evans-Pritchard's narratives about contradictions in Zande thinking (1937). 
But if claims of alternative standards of rationality are to be vindicated, and conversely if cross-cultural study is to contribute at all to contemporary work on rationality, engaging with this abstract work cannot be avoided. When the role of context in rationality is understood, it is easily seen that for all the vividness and all the heat of many evocative cases, such as Zande contradictions, they may have little bearing on the question of alternative standards of rationality. From the perspective of contemporary work on bounded rationality, even the presence of systematic contradictions in the beliefs of a culture may (as I discuss below) be explained rather prosaically. Many of the old debates and examples, while dramatic, are out of date. If cross-cultural work is to provide evidence for pluralism about rationality - as I believe it can - there is no choice but to labor through the dry but careful study of the relation between context and the norms of rationality altogether.

\section{Complexities in identifying reasoning}

Many theorists approach the problem of alternative standards of rationality by observing and characterizing differences in the practices of different cultures, and from these inferring differences in the way they reason and hence the way they think. But even identifying reasoning is much more slippery than many people have assumed.

To make this concrete, consider pyroscapulimancy in the Shang Dynasty. In this widely discussed form of divination, a tortoise shell or the shoulder blade of a cow or buffalo was polished, sawn, and rows of depressions and grooves carved into the underside. The shell or bone was then put into the fire, causing cracking, and the fissures read by diviners or the king. Notations were then made on the shell or bone, listing the question asked and answer given by the reading, and later it was marked on the shell or bone whether the answer been correct. ${ }^{4}$

\footnotetext{
${ }^{4}$ See Chang 1980, Keightley 1988, Smith 1991.
} 


\subsection{What rules of inference?}

One natural way of identifying the reasoning in this case is to take the diviner (i) to have perceived the pattern of fissures of the shell or bone emerging from the fire, forming a belief that the patterns are such-and-such (call this believed proposition $P$ ); and then (ii) to have drawn an inference from $P$ to the conclusion $Q$ about the question being asked. As I will point out in a moment, this is not the only way of describing what is going on in the case. But even if we do understand the inference in this way, we are far from having determined what reasoning takes place. Suppose, for instance, the diviner reasons from $\mathrm{P}$ to $\mathrm{Q}$ using a rule of inference. What rule of inference is used? It may be that the inference is an enthymeme - i.e., that the inference involves implicit premises, such as "if the observed pattern is suchand-such, then the conclusion is so-and-so.” The rule of inference could then be a familiar deductive rule, such as modus ponens. ${ }^{5}$ Or the inference may not be an enthymeme. The inference rule may be more than just a formal rule like modus ponens, but instead a schema that includes the particular case as an instance. Or the inference could be at some level of generality in the middle.

This may seem like splitting hairs, but if the assessment of the rationality of divination in this case involves assessing the diviner's reasoning, then we cannot avoid distinguishing - under the surface if not explicitly - how the diviner reasons from what the diviner believes. If the diviner's reasoning involves a schema from P-like patterns directly to Q-like conclusions, then investigating his rationality will involve considering the rationality of that schema. But if the reasoning is enthymematic as I described, then his reasoning may be no different from a logician's, and questions of his rationality will presumably have to be directed elsewhere, such as at the tacit

\footnotetext{
${ }^{5}$ I.e., from the premises $P$ and $P$ implies $Q$, infer $Q$.
} 
premise. This is but one example of the difficulty in identifying reasoning rules even having taken the drawn inferences as a given. ${ }^{6}$

\subsection{Direct perception versus reasoning}

A second dimension of difficulty is whether and to what extent reasoning is involved at all. Suppose that, on observing a particular cracked tortoiseshell, a Shang diviner arrives at a conclusion, such as that the king should not go hunting on the following day, that a wife of the king will give birth to a son, or that a wine sacrifice should be offered. ${ }^{7}$ What the diviner's observation or perception is may have a substantial impact on what sort of reasoning is involved in arriving at that conclusion. There is a spectrum of views, for instance, as to what sorts of objects we perceive, from the Berkeleyan picture of perceptions as sensible data about which one cannot be mistaken (which nearly no one today believes) to the "naïve" picture favored by many contemporary philosophers that we directly and unmediately perceive ordinary objects. ${ }^{8}$

Even given a particular view on the kinds of things perceived, such as a contemporary version of the "naïve" view, it must be decided which particular ordinary things are perceived, if we are to pin down the reasoning involved. In the case of pyroscapulimancy, the natural answer I suggested above is to take the fissures or patterns of fissures to be the objects of perception. But it may instead be reasonable to hold that the diviner "reads" the conclusion right off of the shell. That is, that he directly perceives the

${ }^{6}$ It also assumes that reasoning proceeds with rules, which is also debated. For an alternative, see for instance Johnson-Laird 2004. This introduces a different dimension of difficulty in characterizing the procedures by which reasoning takes place.

${ }^{7}$ See Tso-pin and Yang 1948: 122-123.

${ }^{8}$ A distinction is also made in the philosophy of perception between the contents of perception from the objects of perception. See Peacocke 1992; Kelly 2007. This distinction alone raises two potentially independent problems in this case, as well as the further problem of what the relation between each sort of content is to the belief formed by the diviner. 
conclusion, rather than perceiving a pattern of fissures and subsequently reasoning to the conclusion. ${ }^{9}$ To identify the reasoning performed by the diviner may require taking a finely detailed position on a theory of perception. ${ }^{10}$

The intersection between issues of perception and reasoning is implicitly involved in a good deal of recent work on both divination and exegesis. As applied to biblical exegesis, for instance, it arises in connection with the question of existence or primacy of the "literal" meaning of the text. It is widely argued that we should not assume that the interpretation of a mystical or allegorical meaning of a text is posterior to or derivative on the simple or literal meaning of the text. ${ }^{11}$ While there are differences between the perception of meaning involved in reading texts and the perception of ordinary objects, similar questions come up as to how we should understand which entities are perceived in both cases. Schofer, for instance, has recently discussed some of these issues in connection with both divination and midrashic interpretation in the Talmud (2005: 6-12). As with drawing inferences in pyroscapulimancy, how much reasoning is involved in textual interpretation depends on what is directly read off the text. If, for instance, the semantic content of the text is already the midrashic reading, then there may be no reasoning process involved at all, and no point to investigate reasoning. Different sets of inferential machinery are involved in inferring an

\footnotetext{
${ }^{9}$ Similarly, when Casca, in Julius Caesar (Act I Scene 3), sees a lion glaring at him and a bird of night shrieking in the marketplace all day long, he does not need to reason to know that they are portents of evil.

${ }^{10}$ Issues of expert deference and justification may also arise in cases of perception, as they do in testimony and other sources of epistemic justification as I will discuss below. Depending on how the diviner's perception is understood, such factors may be involved either in generating or constituting his perceptual content. For new papers on perceptual content, see Hawley and Macpherson 2010.

${ }^{11}$ E.g., Kugel 2007: 10-16.
} 
allegorical lesson from a text if that text is understood to be read literally than it if is understood to be directly read allegorically. ${ }^{12}$

\subsection{Whose reasoning?}

A third dimension of difficulty in identifying the reasoning involved in a practice is the fact that phenomena such as divination take place in social contexts, and the reasoning pertinent to our inquiry into divination may not be the diviner's reasoning at all. All the above problems of perception and reasoning already arise in ordinary inferences performed by a single individual. But in such practices as divination, layers of different people and practices are involved. Some methods of divination involve fairly mechanical steps that may be taken by a lone agent, such as the Zande poison oracle, or qian divination. In qian divination, for instance, a cylinder containing a number of sticks is shaken, and one taken out at random. Each stick corresponds to a given message, so the randomly chosen stick yields a literal if rather generic message. ${ }^{13}$ Iles-Johnston (2005: 299-300) argues that part of

12 This may bear on studies of Talmudic reasoning such as Moscovitz 2002. There may not be a correct answer as to where the lines should be drawn, but it is not hard to imagine that plausible theories of the division of labor could be worked out. In any case, some role should surely be allocated to reasoning in exegesis, while taking it to the extreme that all reading involves inference from perceptual sense-data is putting too much on the shoulders of reasoning. A variety of disciplines are involved with this question, from cognitive science and psychology studying the actual mechanisms of thought. Also pertinent to this is recent work on metaphor and metaphorical meaning, and current theories of linguistic meaning, the role of pragmatics, and the nature of semantic content. See, for instance, Stern 2000 on metaphor, and Recanati 2004 on the semantics/pragmatics divide. Potentially related as well is the issue of objects and texts serving as symbols, as discussed for instance by Struck 2005. One should be careful, however, to avoid inferring that rich perceptual content or symbolic or even mystical meanings are somehow at odds with reasoning. I will discuss this point below in connection with the "symbolist" approach to apparently irrational belief.

13 Smith 1991:238 points out that even qian divination may involve assistance in its interpretation; see also Graf 2005:62. 
the motivation for randomization processes is precisely to eliminate the potential for human intervention and opening the liminal space for divine control. Dice oracles, such as those discussed by Graf (2005), involve similar mechanical procedures. ${ }^{14}$

But in many similar divinatory procedures, the activity and mediation of human agents is not eliminated. Ticketed oracles, described by Frankfurter (2005: 246-249), are performed in a central location, rather than being mechanical procedures that individuals in far-flung locations can perform. And other methods of divination involve many agents in the performance of the act. An incubation or dream oracle may involve a good number of people, such as an oracle and a priest and a supplicant, each of whom may have a part in the interpretation and divinatory reasoning (Frankfurter 2005: 239-243).

Thus it may be inadequate for the theorist investigating the diviner's reasoning to focus on the diviner alone, as opposed to the chain of reasoners among whom he participates. This issue becomes even more prominent when we consider reasoning in the justification of belief.

\subsection{The role of reasoning in justification}

In considering the reasoning involved in the diviner's practices, the entire focus on his own inferences may be misplaced. Supposing that the Shang diviner directly infers $\mathrm{Q}$ from $\mathrm{P}$, the theorist's inquiry into Shang reasoning presumably must involve not only that implicit inference rule, but the reasoning that underwrites or justifies his possession of that rule. The Tylorian treatment of the diviner as a proto-scientist, for instance, is directed more at the question of the justification of his belief than at the question of the reasoning the diviner performs.

Unfortunately, these questions are often elided: the reasoning in justifying or generating a phenomenon fails to be distinguished from the reasoning in

${ }^{14}$ In this form of divination, astragaloi (four-sided cut-off knuckle bones of sheep) are thrown, the results indicating which texts are to be read off. 
the practice itself. In the rationality debates, Beattie (1970: 248) clashes with Jarvie and Agassi (1967/1970: 182-183) on this point. Jarvie and Agassi accuse Beattie of ignoring the question of the rationality of agents in different cultures. They assert that he gives an account of the sociological meanings of magical practices but ignores whether or not agents involved in the practices act rationally, thus sidestepping the entire problem. Beattie responds that the question of the rationality of agents is trivial (particularly on Jarvie and Agassi's minimal view of rationality), and the interesting question is the one of sociological meaning. This may or may not be so, but in any case the mismatch of their respective targets highlights the fact that there are several distinct questions one might ask about reasoning and justification of a practice: (a) the reasoning involved in the performance of a practice, such as the diviner reaching the conclusion, through pyroscapulimancy, that the king should not go hunting on the following day, (b) the reasoning involved in causally bringing about a practice such as pyroscapulimancy being employed in a culture (reasoning which need not serve as the justification for that practice), (c) the reasoning involved in justifying a practice by the participants, and (d) the explanation for why a practice is in place, which may or may not involve reasoning at all. ${ }^{15}$

It is easy to conflate the examination of the reasoning of the diviner [i.e., (a)], with these two other places for reasoning [i.e., (b) and (c)], and to conflate all these with the explanatory project [i.e., (d)]. But each is a separate topic.

Social elements, incidentally, are prominent in addressing the question of justifying or generating an act or practice, even more than they are in the inferential processes involved in the act. The justification of belief is generally a social task, which makes it a complex matter even to identify the justificatory reasoning involved in a phenomenon.

\footnotetext{
${ }^{15}$ My thanks to an anonymous reviewer for help in clarifying this point.
} 


\subsection{Further issues in identifying reasoning}

Yet another dimension of difficulty for identifying the involvement of reasoning has been raised by some theorists who regard phenomena such as divination to be rituals that should not principally be understood as involving belief (e.g., Goody (1961: 158-159)). ${ }^{16}$ If so, then either reasoning is not involved in such phenomena altogether or else it must be understood otherwise than involving the transition from sets of beliefs to new beliefs. There are also more widely discussed obstacles in description and interpretation as well. For instance, in determining the reasoning involved in a phenomenon, the theorist regularly encounters problems such as a paucity of data for interpretation, the difficulty of isolating the phenomenon in question from other phenomena, the trustworthiness of various data sources, the "thickness" of descriptions for correctly characterizing the phenomena, and so on.

\subsection{Reasoning and rationality}

The issues I have mentioned are among those involved even in pinning down or characterizing the reasoning involved in a phenomenon. This also leads one to suspect that when we compare the diviner to the scientist, especially for evaluating whether they have different standards or norms, it is not mainly their reasoning that is at issue. The diviner's reasoning is just one part of the story of how he forms or justifies his beliefs, or chooses his

16 The content of this claim is not straightforward, since 'belief' is often used more narrowly to indicate firmly held commitments, rather than the more general notion used in philosophy (and the present paper) of belief as the attitude we take when we regard some proposition as being true. To deny that these phenomena involve belief altogether is a radical move, and it should not be assumed that treating phenomena such as divination as symbolic, functional, or even part of a distinctive "form of life" implies this. Nielsen 1967, for instance, argues that certain forms of "Wittgensteinian fideism" illicitly do this, separating religious belief from other spheres of deliberation. Others, however, deny that the Wittgensteinian view involves this. See Nielsen and Phillips 2005 and Bauer 2005. 
actions. It is not even clear that it makes sense to speak of standards of reasoning, except as derivative on standards of rationality.

To put it bluntly, the foregoing considerations suggest that, if the goal is to see whether the diviner and the scientist have different "standards," focusing on reasoning is liable to be a red herring. Instead, it is more promising to focus on rationality and the question of the possibility of different standards for what beliefs or actions count as rational. To address this, recent work on the nature of rationality is enormously helpful.

In investigating rationality, the stance taken by much of the recent philosophical literature is modest, fallibilist, and anti-skeptical. It often involves a kind of self-conscious naïvete, not addressing skeptical concerns of reflexivity and power, nor concerns about the integrity or boundaries of cultures or linguistic communities. Rather than seeking certitude, much philosophical work in recent years has focused on advancing our understanding of our own rationality, taking the data on its face. ${ }^{17}$ Ironically, all the work on cross-cultural rationality in the rationality debates had the effect of limiting how deeply people looked at the nuances of rationality even as we conceive of it. With the increased confidence of worrying less about interpretation and considering our own rationality directly, a more nuanced picture of the content and limitations of rationality can be developed.

\section{Scrutinizing Rationality}

In shifting focus away from the question, "does the diviner reason differently from the scientist?" and toward the question "do the diviner and the scientist have different standards of rationality?” it is crucial to give up

${ }^{17}$ A key reason these philosophical innovations have been able to occur is that, in recent years, philosophers have worried less about how to interpret cultures, or to ascribe rationality to them, but rather examined rationality directly. In other words, effort has shifted from the epistemology of rationality to the metaphysics of rationality, reflecting the transition in the philosophy of language from the predominance of Quine in the 1960s through the transitional arguments of Davidson, to the "externalist" views of Kripke and Putnam. 
the caricature of "scientific rationality" as a sort of formal, logical ideal for forming beliefs or choosing actions. In recent decades, theorists of rationality of all stripes have moved away from this picture, largely because of a growing recognition that contextual factors play a crucial role in determining whether a belief or action is rational. I will start with practical rationality, where context-dependence is more obvious, and then apply the same considerations to theoretical rationality. This leads to a point often glossed over in the rationality literature: that cultural factors must also figure into determining the rationality of an action or belief.

Subsequently, I turn to how these bear on the question of whether and when there may be alternative standards of rationality. Based on the work on context-dependence, I begin by objecting to the widely used taxonomy of views of rationality, presented by Hollis and Lukes in the thick of the rationality debates, into conservative, fideist, intellectualist, and symbolist camps. Instead, I propose a different approach to classifying views on rationality, and argue that the best route for defending alternative standards is pursuing a kind of "pragmatic moderate pluralism."

Let me precede this with some key distinctions, with which any treatment of rationality has to begin. The word 'rational' can be applied to many different sorts of things, e.g.,

(1) It is rational to believe that the earth is round.

(2) It is rational to eat a balanced breakfast.

(3) It is rational to draw the inference from It is raining to The ground is wet.

and so on.

Even these simple examples illustrate two key points. First, 'rational' may have more than one distinct meaning (i.e., it may be polysemous), and second, on at least certain meanings of 'rational', propositions expressed by sentences like ' $\mathrm{x}$ is rational' are sensitive to context. 


\subsection{The polysemy of 'rational'}

The polysemy of 'rational' was suggested by Aristotle: sentence (1) is a claim of theoretical rationality, or rationality in belief, and sentence (2) is a claim of practical rationality, rationality in action, intention, or planning. ${ }^{18}$ As for inferences, at least some are normally understood as theoretical, involving transitions from a belief to another belief. There is disagreement, however, about the distinction (and relation) between theoretical and practical rationality altogether. Hume is generally read as holding that the evaluation of ends is not subject to reasons. On this view all rationality is the "instrumental" reasoning about means, ${ }^{19}$ making practical rationality a species of theoretical rationality. ${ }^{20}$ Other theorists have roughly reversed this, regarding theoretical rationality as a species of practical rationality. ${ }^{21}$

And 'rational' may be polysemous in still more ways. Many philosophers understand theoretical rationality to be the same as "epistemic" rationality,

${ }^{18}$ E.g., Nichomachean Ethics 1147a27-28

${ }^{19}$ In some traditions, "instrumental rationality" has come to mean rationality in the service of the exercise of control or power over nature or others. Some theorists are guilty of eliding the Humean sense with this other sense. Ulin 2001:43, for instance, says: "Functionalist anthropology thus operates within a framework of instrumental rationality, that is, as a method whose technical rules of procedure are directed towards the objectification and control of reality.”

${ }^{20}$ Many theorists take issue with this, and some as an interpretation of Hume. See Smith 2004.

${ }^{21}$ E.g., Goldman 1986. 
but in recent years some philosophers have sought to distinguish the two. ${ }^{22}$ 'Practical rationality' too may have several distinct meanings of its own. ${ }^{23}$

The relation between the fact that 'rational' has multiple meanings and the possibility that there are different standards of rationality is a complex one. On the one hand, it seems we need to hone in on just one sense of 'rational', before asking whether there are different standards. In postulating different standards of rationality, the idea seems to be that given one single kind of rationality, such as epistemic rationality or practical rationality, there are different standards for different individuals or cultures. To cast it in this way is to take the Zande healer and the American doctor both to be ascribed the same "epistemic rationality" property, but somehow for the property to be ascribed to the healer on the Zande standard and to the doctor on the American standard.

On the other hand, it may be that the best way of understanding alternative standards is in terms of multiple meanings of 'epistemically rational' or 'theoretically rational'. That is, where we thought that 'epistemically rational' denoted a single property (or relation) across cultures, in fact when we say that the Zande healer is epistemically rational, we are ascribing something entirely different to him than we are when we say that the American doctor is epistemically rational. I will return to this issue below.

\subsection{The context-dependence of rationality}

Apart from the possible polysemy of 'rational', the three numbered sentence examples above may be context-dependent in various ways. An

\footnotetext{
22 E.g., Kelly 2003, Harman 2004.

${ }^{23}$ Some theorists advocate "internalist" or "externalist" theories of rationality, while others leave room for both. And as I will discuss below, ideal rationality may be separated from rationality as restricted to various constraints or bounds, or rationality as restricted to different domains of application. Cf. Stein 1996, Samuels et al. 2002, Samuels et al. 2004.
} 
obvious way that a claim of practical rationality depends on context is that an act's instrumental rationality depends on the ends of the actor. Consider, for instance, sentence (2) as applied to various contexts. It is normally true when asserted about an ordinary person who wants to have a productive day, but it is false if asserted of a person wishing to continue a hunger strike, or preparing for a glucose test. If my overriding aim is to be alert for the day then (2) is true, but if my aim is to obtain an accurate reading on my blood glucose test, it is not.

The proposition expressed by sentences such as (1) may or may not depend on various contextual factors as well. Consider for instance, Anaxagoras's young son believing that the earth is flat. Even though the boy's belief is mistaken, it is common to regard the boy as being rational in deferring to his father, since the boy is not a scientist and can generally rely on Anaxagoras's claims, and moreover Anaxagoras had good arguments for the flatness of the earth. ${ }^{24}$ The same, however, might not be said of a contemporary flat-earther, who is aware of but ignores the overwhelming evidence that the earth is round.

Whether a belief or action is rational depends on context. The rationality of an action must be evaluated in part against the background of the ends of the actor, and the rationality of a belief in part against the background of the knowledge possessed in the believer's community. Does this serve as ammunition for the rationality pluralist? Or is it ammunition for her opponent? Does it strengthen or weaken the case for the Zande healer having a different standard of rationality than the American doctor? To make progress on this, we will need more clarity on the many different ways rationality can depend on context.

As the next several pages make clear, rationality depends on context in so many ways that the rationality pluralist may be inclined to throw up her hands in surrender. Current approaches to rationality have grown sophisticated enough that it is now taken as obvious, among theorists, that different people

\footnotetext{
${ }^{24}$ Anaxagoras DK59 A42.
} 
in different contexts can and should depart from "ideal rationality" in different ways. This makes it a much more nuanced matter to see if and how there can be alternative standards keyed to cultural difference.

In the end, I will suggest that there remain plausible and moderate ways to understand alternative standards of rationality. From the perspective of the study of religion and ethnography, however, the first result of studying the context-dependence of rationality is that nearly all the old-school arguments for alternative standards fail, having been trumped by more careful work on the idea of rationality. As I will discuss shortly, this work shatters the commonly used taxonomy of approaches to rationality, dividing views into “conservative," “intellectualist," “fideist," and "symbolist” positions. Inasmuch as theorists identify with one or another of these categories, or use them to structure their thinking about rationality or reasoning, their claims are built on a discredited foundation.

\subsection{Moving past the ideally-rational/irrational dichotomy}

Challenges to rational choice theories of action have been a central topic in psychology and economics in the last few decades, and in recent years (particularly since the recent financial crisis) it has been receiving popular attention as well. ${ }^{25}$ The leading theory of rational choice is expected utility theory, ${ }^{26}$ and some robust psychological evidence seems to show that people systematically violate what this theory would predict, and hence routinely and systematically have inconsistent ends. ${ }^{27}$ For instance, people show a bias for

${ }^{25}$ Some books on the topic have recently hit the bestseller lists, including Ariely 2009 and Thaler and Sunstein 2008. In psychology, this is represented by the heuristics and biases literature associated with Kahneman and Tversky and work on bounded rationality initiated by Herbert Simon.

${ }^{26}$ Von Neumann and Morgenstern 1944.

${ }^{27}$ Some theorists (e.g., Camerer and Thaler) assume that even in these cases "underlying” preferences are consistent, while others consider the possibility that agents do have inconsistent preferences. My thanks to Robert Sugden for bringing this to my attention. 
keeping things they already possess. If a person is given $\$ 200$, she may be unwilling to use it to buy a Springsteen ticket, but if the same person is given a Springsteen ticket, she will be unwilling to accept $\$ 200$ for it. If expected utility theory correctly characterizes the norms of instrumental reason, then this evidence shows that the ticket-buyer/holder acts irrationally. A common way to characterize this (particularly in popularizations on the topic) is that economists mistakenly believe that people are rational, and that experimental work has shown that people are actually irrational.

The assumption behind this characterization is that rationality is the same as ideal rationality, which in turn is correctly modeled with some theory of rational choice such as expected utility theory. There is, of course, a different response, namely that expected utility theory does not correctly capture the norms of rationality. This may either be because it is not the correct theory of ideal rationality, or else because it is incorrect to equate rationality with ideal rationality.

A growing literature accounts for apparent irrationalities in this last way. Instead of taking rationality to be an ideal, what actions count as rational depend on what good strategies are for making decisions in the context, i.e., they depend on the "bounds" imposed by resource, time, informational, or attention constraints. ${ }^{28}$

${ }^{28}$ Compare, for instance, two different games of chess played by Gary Kasparov, one an untimed game and one a game of speed chess. In playing speed chess, he makes different moves, and uses a different decision procedure, than he would use in an untimed game. With the board in a particular configuration in an untimed game, he makes move $\mathrm{A}$, whereas in speed chess he makes move $\mathrm{B}$. The reason is easily given: because of the time limits of speed chess, there were constraints on how well he could optimize. Because of the costs of taking too long for the choice, using a more accurate decision procedure would have produced a worse outcome. We might characterize Kasparov's choice as irrational, as failing to arrive at the ideal choice. Or instead, we might regard him as an ideal agent but subject to time constraints, or as acting as a bounded rather than ideal rational agent. Many approaches to this are discussed in Manktelow and Over 1993, Evans and Over 1996, Preyer and Peter 2000, Elio 2002, Mele and Rawling 2004, Leighton and Sternberg 2004. 
Another potential factor in determining the practical rationality of an action is the domain in which an action takes place. In the domain of court stenography, there may be different norms for how carefully one should type, as compared to typing in the domain of texting a friend from a concert. The demands imposed by the domain in general may figure into which actions count as rational as applied to that domain.

These examples of bounded rationality have become an integral part of the thinking of contemporary theorists of rationality. The theorist of rationality, on encountering a cultural difference of any sort that leads to a systematic change in what actors do - whether it is choosing to eat or refrain from eating breakfast, or choosing to speak to or refrain from speaking to the medicinal bark - will naturally regard it just as this sort of domain change.

This is a key reason that in the flurry of recent work on rationality, little attention has been paid to cultural difference. We already encounter countless different domains and different bounds in everyday life, so there seems to be nothing special about a few more.

If we want to situate spheres of practice such as science, religion, or divination in rationality, we must determine whether they are domains in this sense to which the rationality of an action is to be relativized. And again, if so, what that implies about standards of rationality: does contextualizing rationality to these different domains imply that that there are or are not different standards?

\subsection{The same issues apply to theoretical rationality}

It is not only actions whose rationality depends on context. Many of the same considerations that affect the rationality of actions under various circumstances and constraints also affect the rationality of belief. ${ }^{29}$ Here too

29 Psychological experiments have demonstrated that people regularly commit the conjunction fallacy, the gambler's fallacy, base-rate neglect, violations of modus tollens, overconfidence, belief perseverance, anchoring, and so on. Rysiew 2007 provides an excellent overview of these and overview of recent debates on rationality in psychology and epistemology. 
an active program in rationality research involves accounting for these apparent violations not as irrational but instead as good strategies for forming beliefs under realistically limited conditions.

As with the dependence of practical rationality on ends, the theoretically rational depends on context in obvious ways as well. What is rational to believe, for instance, depends on the information one already has and is given. Depending on what evidence he has besides his father's testimony, Anaxagoras's son would plausibly be irrational to believe that the earth is round. ${ }^{30}$ The fact that we live in very restricted informational situations may even be a deep part of what counts as epistemically rational in the first place. A perfect epistemologist, perhaps, is an omniscient one - but of course, if an epistemologist is omniscient, then she already possesses all true beliefs, so there is no generation of true beliefs to be done. Any norms of epistemology are principles for at least partly ignorant knowers. And plausibly, what the norms are will need to be adjusted, depending on the ways in which the knowers are ignorant.

Even logic is not immune. In the face of non-ideal circumstances, the rational agent might be well advised to compromise the most cherished logical and epistemic principles. In the rationality debates, the context dependence of norms of rationality was often obscured by focusing on putative violations of the rule of noncontradiction (such as Zande thinking about "witches" and inheritance). Irrationality was often treated as interchangeable with violations of logical rules. But this is a mistake: violating logical strictures does not necessarily indicate irrationality. Following a defeasible rule of thumb may be better than following a logical rule, depending on the costs and resource requirements involved in following

${ }^{30}$ A critical issue bearing on this is the possibility of divergence between being justified and being epistemically blameless. It is a subtle matter to tease these apart; see Goldman 1980; Plantinga 1993. If they are distinct, the correctness of this and subsequent points will depend on pinning down how the rationality of a belief is related to each of these. 
the rule. ${ }^{31}$ Even if there were agreement on a single logical system that best captured the universal rules of reasoning - which there is not - following such rules is neither necessary nor sufficient for rationality. Supposing the Azande did hold beliefs that were mutually contradictory, this could reflect epistemic virtue on their part, rather than epistemic deficiency. A good deal of the force of such examples in the rationality debates was predicated on overestimating the role of logical reasoning and conformance to the rules of classical logic in belief formation in general.

The rational agent might also be well-advised, depending on circumstances, to compromise even epistemic principles that are plausibly justified a priori. Perception, memory, reason, and testimony characteristically yield rational beliefs. But depending on circumstances, forming new beliefs may be more costly than it is worth. Suppose we have limited memories, so that the acquisition of a new belief forces us to forget an old one, and suppose we have reason to believe that all our old beliefs deliver more truths than new beliefs would. If so, it may be rational to avoid forming new beliefs even on the basis of these sources.

\subsection{Moving to cultural contexts}

Most work on the context-dependence of rationality focuses on bounds on the agent, such as information, time, resource, and attention limits. Less often considered are the constraints imposed by the cultural context in which the

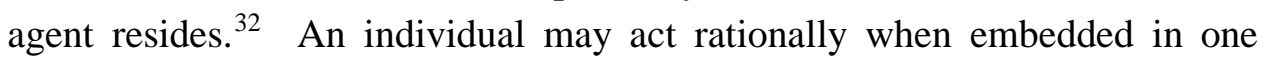
culture, and yet without changing her beliefs or knowledge or intelligence or

${ }^{31}$ Kyburg 1987, Pollock 1991, and Nozick 1993 argue that it is not always irrational to be inconsistent. An alternative, of course, is to model inference with a different logic. There is a large literature on logics that accommodate inconsistency, and some attempts to apply such logics to the Azande in particular. See, for instance, Cooper 1975; da Costa and French 1995.

32 Here, as above, I am employing an at least modestly externalist conception of rationality. 
domain of interest, may be acting irrationally when embedded in a different one.

The most obvious way that norms of belief or action depend on culturewide factors is through deference to experts. If I want to quench my thirst, it is rational for me to drink from the tap, and irrational to drink from the Charles River. The reason it would be irrational for me to drink from the Charles depends on an enormous set of factors of which I am quite unaware, including water studies and engineering processes and chemical analyses and networks of urban infrastructure. Not only do I not know the results of the studies and the nature of the processes, I do not even know what kinds of things would be needed to justify my attitude toward water in the Charles. The reason drinking from the Charles is irrational for me is not a result of my knowledge, but of the knowledge of others in my community.

Simply the fact that some piece of information is easily available in my culture may change the actions that are rational for me, whether or not I am aware of it. Even if I know nothing about retirement vehicles, it can be irrational for me not to sign up for a 403(b) account. The fact that I know nothing about the advantages or disadvantages of signing up may not excuse my irrationality, since to be a rational actor not only involves being open to one's senses or passively hearing the testimony of others, but also making the appropriate efforts to find out what is widely known. ${ }^{33}$

Many factors that affect the rationality of action on an individual level have analogues on a cultural level. Given the ability to make use of others in the community, the rationality of an action or belief for an individual may depend on community-wide conceptual resources, informational constraints, resource constraints, technological constraints, time constraints, attention

${ }^{33}$ This gives rise to fascinating problems in cases of cultural collision, such as those described in Fadiman 1997 on the encounter of Hmong immigrants to California with the American medical system. It vividly demonstrates the extent to which norms of deference come in conflict with one another, and how individuals may find themselves deferring inconsistently, even by their own lights, because of the complexities of navigating the conflicts between deference to different sets of experts and traditions. 
constraints, and so on. And also domains: the rationality of some action an individual takes in building a house, for instance, may depend on how various types of construction work are hierarchically organized in the community or culture.

Further, the ends or goals of a culture may be among the most important contextual parameters for determining the rationality of an action or belief. The reliance on the social implies that ends and social functions may cascade into the rationality of individual action. If there is a structural or culture-wide end of maintaining stability, or keeping lower castes in their place, the rationality of an individual action may depend on (and hence operate in service to) such ends. Properly contextualizing rationality thus has the potential to blur or even erase the line between, for instance, rational choice and functionalist explanations of human action.

\section{Views on alternative standards: A flawed taxonomy}

The basic categories for organizing approaches to cross-cultural rationality need to be rethought, once we observe the variety of ways contextual factors - especially the cultural ones mentioned in the previous section - can figure into ascriptions of rationality. Consider the taxonomy in which Hollis and Lukes fit alternative approaches in Rationality and Relativism: $:^{34}$

\footnotetext{
${ }^{34}$ Hollis and Lukes 1982: 12.
} 


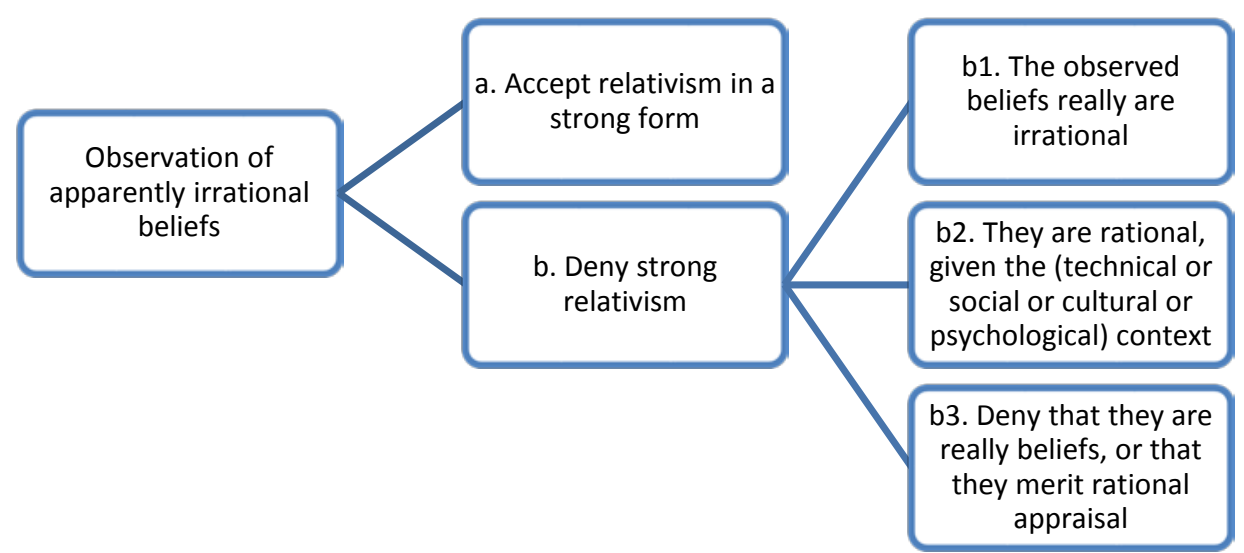

When we observe an apparently irrational belief, such as the Zande healer's belief that speaking to the bark is connected with predictive belching, the taxonomy seems to present a comprehensive set of alternative reactions. Hollis and Lukes divide the reactions into relativist and non-relativist ones, and then break down the non-relativist ones into further subcategories. Box (b1) - the conservative position that the healer's belief is simply irrational - is a position most theorists distance themselves from, taking it to be condescending, ethnocentric, or simply insufficiently explanatory, though some people such as Hollis (1982) and Boghossian (2006) take a view along this line. The other three categories are generally associated with particular theorists:

(a) is associated in the rationality debates with Winch's interpretation of Wittgenstein, and with relativist philosophers of science (e.g., Barnes and Bloor 1982). Some theorists call this view "fideism," following Nielsen's paper (1967) on Wittgenstein, while others seem to use the term differently. Related approaches are also advanced by antirealists such as Goodman (1978), Rorty (1998), and Putnam (1990).

(b2) is associated with Tylor's position that phenomena such as divination represent the pursuit of scientific goals in more limited or different epistemic contexts. Horton (1993) is the primary representative of this position, often called "intellectualism," in the rationality debates. 
(b3) is associated with views that take apparently irrational beliefs to be "expressive" or "symbolic," typically in a non-cognitive mode. Theorists advancing such views include Leach (1954), Goody (1961), Beattie (1964; 1966), and Firth (1964). This category is also associated with views that demote the role of individual agency in favor of social or structural explanations, and so functionalist theories may fall in it as well.

This taxonomy is echoed in a number of places. ${ }^{35}$ In the abstract, it seems to be a useful way of parceling out the possible positions. On closer scrutiny, however, this conservative / fideist / intellectualist / symbolist taxonomy is unworkable. Box (b2) in particular is an enormously flexible position. The "intellectualist" variant of (b2) holds that "the general purpose of belief systems...is to explain, predict, and control the world," and "imports out categories such as science and magic and puts them to work in analyzing other cultures" (Simon 1998: 84). Horton (1967), for instance, regards the quest for explanation as being among the most fundamental of human pursuits, and recognizes that different explanations are appropriate in different informational contexts. Yet whatever the plausibility of "intellectualism," it is only a special subcase of (b2). Box (b2) does not even specify the kinds of contextual parameters of 'rational', and certainly is not limited to the specification of the ends of rational inquiry as being a certain kind of explanation or the control of nature. Identifying (b2) with the "intellectualist" position is thus misleading.

Depending on how functionalism is understood, it too may fall within (b2). Even culture-wide factors may reverberate in what is rational for an individual, because of the social dimensions of epistemic deference and epistemic norms. Moreover, there are a number of ways the functional explanation of an agent's action or belief may be compatible with the action

${ }^{35}$ For a recent overview of rationality and cultural relativism following this structure, see Simon 1998. Tambiah 1989 also applies a similar division. 
or belief being rational relative to the context in question. ${ }^{36}$ And even on a more standard account of rationality, if agents pursue actions with the aim (explicit or implicit) of performing a stabilizing function for society, the actions they take may even be ideally rational. ${ }^{37}$

Finally, even boxes (b3) and (a) may not be distinct from (b2). As I pointed out in discussing perception above, the question of the "meaning" of a phenomenon may be quite independent of the question of the reasoning involved in it. And the contextual factors in (b2) may encompass everything that the fideist takes to be relevant to relativizing rationality. Depending on what cultural and social factors are included, even the "forms of life" to which the Wittgensteinian advocates relativizing rationality may be accommodated within (b2).

To give just one example, consider Beattie’s “symbolist” view. He holds that a ritual or magical rite "has an essentially expressive aspect, whether or not it is thought to be effective instrumentally as well... I am not saying that ritual and magical activities are not commonly thought to be causally effective; they certainly are. But they are expressive as well as being instrumental, and it is this that distinguishes them from strictly empirical, instrumental activity.” (1964: 203-4)

In contrasting symbolic/expressive aspects of an activity with empirical/instrumental aspects, Beattie assumes a narrow view of

${ }^{36}$ On a teleofunctional account of rationality, the compatibility may be nearly trivial. Cf. Dretske 1981, Millikan 1984. These views typically focus on the function and survival of individuals, but are compatible with group and social functions as well.

37 Robert Sugden has pointed out to me that on standard rational choice theory, it is typically irrational to concern oneself with "social functions" in anything but very small societies, because of the free-rider problem and the impossibility of any individual having a substantial impact on a large society. It would be rational if support of the social function were one's only end, but with plausibly self-directed ends as well, they would likely overwhelm the social ends in the determination of rational action. Sugden also points out that on other accounts of rationality, such as rule-consequentialist and team-reasoning accounts, this objection may be surmounted. 
instrumental activities, taking them to be just causal means for bringing about material states of the world. Merely by broadening the notion of an "end" to include symbolic or expressive aims, and correspondingly broadening the notion of an "instrument" to include activities directed at achieving such aims, this view becomes a subcase of (b2). Moreover, by including properties of the community or culture among the factors to which rationality is contextualized, these aims need not be possessed by the actors themselves, but may be implicit aims of the community.

The Hollis-Lukes taxonomy has been influential, and seems to be a sensible carving up of possible approaches. But in fact the divisions between conservative, fideist, intellectualist, and symbolist are not very useful. A wide range of positions - and arguably, all the interesting ones - fall into the various ways rationality may depend on context. The association of box (b2) with the "intellectualist" position, and the appearance that Hollis and Lukes have distinguished this box from the others, is only a consequence of their having left it unclear how contextual factors are to be understood.

Whether one intends to challenge or defend rationality pluralism, the Hollis-Lukes taxonomy does not help stake out the different positions one might take. Instead, we need another way of contrasting and assessing the plausible views.

\section{A different approach}

The traditional or orthodox position on rationality - that there is one set of demanding rules a rational agent must follow, such as "Western rationality," whatever that might be, or such as the axioms of expected utility theory - is not easy to maintain. This might seem to spell an easy victory for the advocates of alternative standards of rationality. But in fact, the same considerations that undermine traditional conceptions of rationality also make life difficult for the fan of alternative standards.

When the received view was the orthodox position, it was easy to oppose it with a relativist view on standards of rationality. But in light of the 
considerations above, the opposing position is murkier. Consider the following pairs of claims:

(4a) It is rational for the diviner to ask the bark to make him belch.

(4b) It is not rational for the scientist to ask the bark to make him belch.

(5a) It is rational for the person aiming to be alert to eat a balanced breakfast.

(5b) It is not rational for the person aiming to have an accurate glucose test to eat a balanced breakfast.

Suppose we want to defend the truth of both (4a) and (4b), by relativizing rationality to the cultural contexts in which the diviner and scientist are respectively embedded. And suppose that in doing so, we assert that the diviner and the scientist have "alternative standards" of rationality. Would this move force us into the same treatment of (5a) and (5b)? Can a wedge be driven between the pairs of cases, so that (4a) and (4b) do exhibit alternative standards, while (5a) and (5b) do not?

In this question, the entire "alternative standards" project is at stake. For there to be alternative standards, it cannot be as easy to change one's standard of rationality as it is to change one's mind or change one's socks. Otherwise, rationality pluralism is trivialized.

To begin making sense of the idea of alternative standards of rationality, I propose a different and simpler way of dividing up views on rationality. Taking as a starting point that rationality should be divided into different "genera," such as epistemic rationality, theoretical rationality, etc., within each "genus" let us divide views into what we might call monist and pluralist ones. Monism about a genus of rationality takes it to consist of just one property (or relation), while pluralism takes a genus to consist of many different properties (or relations).

The claim that there are alternative standards of rationality, I will suggest, amounts to holding a moderate form of pluralism. Neither any sort of monism nor an extreme pluralism will be consistent with there being alternative standards. Only if we can make sense of moderate pluralism will alternative standards even be possible. 


\subsection{Understanding monism: a brief formal treatment}

Intuitively, the idea of monism is that a genus of rationality is "just one thing." Epistemic rationality, for instance, is one property or relation, the same when ascribed to one belief as when ascribed to another. It might be thought that this is an orthodox or inflexible view. But this is a misperception. In fact, monism is so flexible that nearly all contemporary theorists - even the most liberal about rationality - are monists of some sort or other.

The most familiar sort of monism is one I discussed above, the view that there is one demanding set of rules or conditions that a belief or action must satisfy to be rational, whoever the conceiver and whatever the context. This view, which we might call traditional or orthodox monism, is indeed rather inflexible. And as I pointed out above, it is not likely to be viable. What were once held to be the main candidates for "the rules of reasoning," such as theories of implication and consistency, are simply not the same as the guidelines a reasoner should follow in order to form beliefs or choose actions. $^{38}$ Orthodox monism faces irreconcilable demands on what the unitary rules should be.

Notice that even orthodox monism relativizes the rationality of an action to at least some contextual factors, such as to ends. Accommodating some context dependence is required for any view of rationality to be tenable. ${ }^{39}$ The reason monism is such a flexible category of views is that it is open to the monist to relativize rationality to as many contextual factors as she likes.

The way a monist typically accomplishes this is to understand rationality as a relation between beliefs (or actions) and other things, and not as an

\footnotetext{
${ }^{38}$ See also Harman 1995 and 2004.
}

${ }^{39}$ A view that denies, this, e.g., taking a claim such as "It is rational to eat a healthy breakfast” to be either true or false simpliciter, is entirely untenable - we might call it wing-nut monism. 
intrinsic property of beliefs (or actions). ${ }^{40}$ A simple way to illustrate this is by considering how the monist might treat 'rational' as a many-place predicate. Superficially, the structure of sentence (2), "It is rational to eat a balanced breakfast," would appear to suggest that its logical form is:

(2’) practical-rational(eat-a-balanced-breakfast)

This representation, however, does not reflect the obvious dependence of the truth or falsity of (2) on the aims of the actor. The default way to accommodate this is to take 'practically rational' instead to be a tacitly twoplace predicate, i.e., a name for a relation between actions and ends, rather than just naming a property of actions alone. The following, then are more perspicuous representations of (2):

(2a') practical-rational(eat-a-balanced-breakfast, be-alert)

(2b’) practical-rational(eat-a-balanced-breakfast, have-an-accurateglucose-test)

With this treatment, the value of the second parameter may be picked up by context, and whatever context (2) is uttered in, the predicate 'practically rational' has only one meaning, denoting a single relational property between an action and an end.

Naturally, this treatment can be applied to other contextual factors, besides ends. Which makes monism an extremely flexible view: there is, in fact, no formal obstacle to including as many parameters as one likes into the relations practically-rational, epistemically-rational, etc. Even the most liberal advocate of innumerable contextual factors can be a monist about rationality - i.e., can hold that there is just one single relation for each genus of rationality, across all cultures and contexts.

${ }^{40}$ Similarly, the monist can take rationality to be an extrinsic property of beliefs (or actions), with the holding of the property being taken to depend on a variety of external factors. Or she may also choose to relativize truth, as is becoming fashionable in some quarters in the philosophy of language. 


\subsection{The serious challenge posed by monism}

With just this much formalism in mind, we can articulate what is perhaps the most popular view on rationality nowadays. This view, which we might call "liberal monism," is the crucial challenge to any advocate of alternative standards of rationality. Applied to the practical rationality of actions, the liberal monist view is often cast as holding that there is a prudential "instrumental principle” governing action: take effective and available means to your ends.

A variety of contextual factors may figure into an action being an "effective” means, including the knowledge, capacities, resources, cultural features, and so on. Likewise, a variety of contextual factors may figure into an action being an "available” means. Thus practical rationality not just a property of an action, or a relation between an action and an end, but a multiplace relation between an action, an end, and all the contextual factors that figure into that action being "effective" and "available" means to the end.

The liberal monist will apply some rather general norms to what counts as effective and what counts as available. If an agent knows that the best course of action for her is to file her taxes, knows that she will suffer dire consequences if she does not, and wants to file her taxes, but nonetheless fails to because she's lazy, the liberal monist will generally count this as irrational (in particular, as a case of akrasia, or weakness of will). On the other hand, if she has a psychological block of some sort that makes it extremely difficult for her to fill out forms, then her failure to file might count as rational, since filing might not count as an available course of action for her. On the liberal monist view, some factors, such as psychological impairment, are eligible to adjust whether an action counts as rational. Other factors, such as laziness, are not. The more liberal the monism, the more accommodating the norms of “effectiveness” and “availability.”

Applying this to the Zande healer and the American doctor, the liberal monist will diagnose the systematic differences between their actions as applications of the same instrumental principle in different contexts. On this view, there is no need to postulate any difference at all between the standard 
of rationality of the Zande healer and that of the American doctor. Both take "effective and available" means to their ends, whatever those ends are. What is available to the Zande healer differs from what is available to the American doctor, and moreover their ends may differ and what is effective may differ as well. But the principle is the same.

The liberal version of monism is the greatest threat to the rationalitypluralist. But there are still more positions available to the monist. Taking the contextualization of rationality to an extreme, there is a perfectly consistent view that remains monism, but whose effect is the polar opposite of traditional monism. Instead of insisting that there is one demanding set of rules that every action or belief must satisfy, it allows the one rationality relation to be adjustable enough that the rationality of an action or belief is relativized to every element of context. This approach we might call hippie monism.

Hippie monism is not likely to be an attractive view. If rationality is truly adjusted to every contextual factor, it effectively vaporizes the normativity of rationality altogether by making it apply to every action or belief - tout comprendre c'est tout rationaliser.

The point, however, is that monism about rationality is much more flexible and robust than many have assumed. Extreme versions of monism about rationality, such as orthodox monism and hippie monism, are likely to be unacceptable. Yet there is a large spectrum between the extremes. It remains to be seen whether a satisfactory moderate form of monism can be constructed. But at the very least, there are a great number of ways a theorist can hold that there is just one single relation for each genus of rationality, across all cultures and contexts.

To take stock so far: the rationality pluralist encounters a resourceful opponent, in the contemporary monist. Wherever the ethnographer tracks down radical and systematic differences in the beliefs or practices of other cultures, the monist can generally accommodate these differences in a moderate form of monism. Whether the phenomenon is pyroscapulimancy among the Shang or the embrace of contradictions among the Azande, the 
monist prefers to introduce yet one more parameter, release yet one more pressure valve, to preserve her monism and deny that the phenomenon reveals the presence of alternative standards.

\subsection{Creating a space for pluralism}

A pluralist view takes there to be different rationality properties or relations, employed in rationality claims made in different contexts. Instead of representing (2) as (2a') in one context and (2b') in another, on a simple pluralist view they might be represented as:

(2a*) practical-rational-to-be-alert(eat-a-balanced-breakfast)

$\left(2 b^{*}\right)$ practical-rational-to-have-an-accurate-glucose-test(eat-a-balancedbreakfast)

In (2a') and (2b'), a single two-place relation practical-rational(_,_) is claimed to hold of an action paired with an end. In contrast, in $\left(2 \mathrm{a}^{*}\right)$ and (2b*) two different one-place relations practical-rational-to-be-alert(_) and practical-rational-to-have-an-accurate-glucose-test(_) are claimed to hold of an action alone.

The intuitive idea of pluralism is that it is mistake to consolidate all the varieties of rationality into one, highly variable and context-dependent, relation. Instead, a genus of rationality, such as epistemic rationality or theoretical rationality, is "more than one thing." 41

The pluralist, however, must be careful to avoid sliding into the view that rationality is innumerably many things. Just as hippie monism is tempting to the liberal monist, an analogous position is tempting to the pluralist. Facing the challenges from monism, it may be tempting for the advocate of

${ }^{41}$ A difficult issue arises in connection with any pluralist view. Suppose we do conclude that in two different contexts the statement "Action A is rational" is to be understood as involving two different predicates entirely. Then it is not clear in what sense there is a disagreement, i.e., in what sense they are to count as alternatives, as opposed just to referring to two different and unrelated properties. I believe this issue can be addressed, but I will leave it to the side for our purposes. Similar questions are discussed in the "relative truth” literature. See Garcia-Carpintero and Kölbel 2008. 
alternative standards to see alternatives everywhere. "Not only do the diviner and the scientist have different standards of rationality," the pluralist may say, "but each and every one of us has alternative standards." My norms of rationality are different from your norms, and those are different from hers, and from his. Instead of including every feature of context as a parameter of a single rationality relation, the hippie pluralist takes every difference in context to ground a different rationality property entirely. There is a certain appeal to this - why should what counts as rational for me also count as what is rational for a different person?

Like hippie monism, however, hippie pluralism is implausible. It generates the result that not only are rational-among-contemporaryAmericans and rational-among-Shang distinct properties, and not only rational-for-Einstein and rational-for-Palin, but also rational-for-Einstein-at2:00pm and rational-for-Einstein-at-2:01pm. With a plenum of different senses of 'rational', the notion is again emptied out.

For a version of pluralism to be tenable, we have to abstract, at least to some extent, over contextual differences. That is, we have to allow ourselves to take 'rational' to denote the same relation across some different individuals and some different contexts. If not, we can never be in a position to contrast their standards of reasoning at all.

\subsection{A promising moderate pluralism}

Consider what we mean by "alternative standards" in mundane contexts. For certain “gradable” adjectives, such as ‘tall' and 'rich', context is involved in resolving their standards or gradations. For instance, Shaquille O'Neal is tall when assessed in the context of humans, but not tall when assessed in the context of all objects seen on $34^{\text {th }}$ Street in Manhattan.

In unpacking the claim Shaquille O'Neal is tall for a human, but not tall for an object on $34^{\text {th }}$ Street, it is common to say that the clauses 'for a human' and 'for an object on $34^{\text {th }}$ Street' set different standards for height. But this terminology elides an important distinction. If a standard varies continuously with variations in the standard-setting bits of context, then in a crucial sense 
we do not have a standard at all. The intuitive idea of alternative standards is more appropriately applied when a predicate does not vary continuously, but has stable clusters and varies only in discontinuous jumps or chunks.

Whether bits of context make a predicate vary in a continuous or in a "jumpy" way is normally invisible from a formal perspective. But it may be just how the predicate depends on contextual parameters, e.g., whether it jumps from stable cluster to stable cluster, rather than varying continuously, that determines whether it denotes different relations across certain contexts. We may not want to distinguish alternative "standards" whenever there are different clusters. For instance, there may be differences between what is rational for the blacksmith and for the silversmith, or between what is rational for the veterinarian and for the tax attorney, in their respective domains. And yet we may choose to abstract over these differences, because it may only be radical cultural differences that are appropriate to the theoretical purposes.

On this approach, determining alternative standards is a pragmatic matter. There is not likely to be a single answer to whether there are different species of rationality, but only answers as to whether there are substantial differences across dimensions of interest.

Perhaps the most interesting of the dimensions is the conceptual resources available to a culture. Despite Davidson's strenuous attempts to rule it out (1973), recent work in the philosophy of language and thought is poised to reopen the possibility that there may be systematic differences in the conceptual resources available to different communities. ${ }^{42}$ If there is a significant sense in which the "space of conceivability" can be understood to differ between the Zande healer and the American doctor, there may be grounds for distinguishing the norms for operating within each respective "conceptual space."

A strong caveat must accompany this, however. As crude as our understanding of rationality is at present, our understanding of concepts and conceptual resources within and across cultures is even more so.

${ }^{42}$ Seeds of this may be found, for instance, in Haslanger 2005, Epstein 2010. 
Demonstrating such conceptual differences, if there are any, remains no small matter. And even supposing there are such differences, it is not clear that they should correspond to significant differences in the actions or beliefs that count as rational. If this is a promising avenue for grounding rationality differences, it will only come with a deeper understanding of the concepts and "conceptual fields" that we and others possess. Though it may be the most interesting potential source of alternative standards of rationality, other dimensions along which there are "jumps" and "stable clusters" remain more likely.

This "moderate pragmatic pluralism" is only a schematic program for reintroducing an interesting notion of alternative standards for rationality, after the recent proliferation of liberal and sophisticated monist approaches to rationality. How those pragmatic divisions are to be forged is a question for the joint engagement of the same fields that had been party to the rationality debates a generation ago - ethnographers, philosophers, religion scholars, cognitive scientists, and so on.

\section{The diviner and the scientist}

Does the diviner have the same standard of rationality as the scientist? If not, is the rationality of one of the two defective?

In the rationality debates, alternative standards were often discussed in connection with people and cultures residing in "different worlds." The idea seems to be that in different cultures, we find differences between the norms of rationality, which mirror differences in the ontologies or the "furniture of the world" for those cultures. Yet it was never clear how the explanation was supposed to run. Are cultures are supposed to have different ontologies in virtue of differences in their respective norms of rationality, or have different norms of rationality in virtue of having different ontologies? But no matter: neither direction seems promising. There may be some relation between the nature of institutions and other socially constituted entities and the norms of

rational action or belief in a context. But it is not clear why the divisions 
between different "standards" of rationality - if there are such divisions should align at all with differences between ontologies - again, if there are such divisions.

To be sure, Levy-Bruhl drew ontological implications from the claim that certain cultures are "pre-logical," but as we have seen, his picture of logic and reasoning was a caricatured one. And even if there turns out to be some way of making sense of "conceptual scheme" difference, whether along the lines of Quine's webs of belief, Wittgenstein's forms of life, Foucault's epistemes, or some other, none of these gives any clear reasons for associating them with a corresponding set of norms for rationality or rules for reasoning.

The bulk of this argument has been a cautionary one. The work in recent years on the contextualization of rationality may seem to support alternative standards, inasmuch as it has challenged the traditional orthodoxy. But as I have argued, it actually gives monism enormous resources, given any genus of rationality, to assimilate all differences into a unitary notion. Thus at least as much as before, the mainstream view on rationality tends to reject the view that there are alternative standards. This mainstream view deserves careful attention, since advocates of different standards often underestimate the changes from the orthodox to more contemporary approaches to rationality and ignore the considerations in favor of monism.

And yet, there is reason for optimism that on balance, the best view will end up being a form of moderate pluralism. The arguments for monism tend to rely excessively on formal considerations, which allow all different contextual factors to be treated equally, as opposed to considering the substantive grounds for dividing the properties and relations we use from one another. Not just any pragmatic factor is adequate to ground distinct species within a given genus of rationality. But where there are significant and systematic culture-wide differences between cultures A and B - and especially when they are systematic differences between conceptual resources, overall aims, large-scale belief systems, etc. - these may be pragmatic grounds for taking A's species of a particular genus of rationality to be distinct from B's species. 
Still, the caution remains. The scrupulous cultural theorist needs to be aware that no matter how different an agent's behavior in some remote context, such differences are far from enough to demonstrate differences in the reasoning the agent employs. Even identifying the reasoning and the heuristics employed in a given situation is a difficult matter. And then, even if differences in reasoning have been identified, the theorist is still a long way from having demonstrated that there are differences in the standards of rationality to which the agent's reasoning is subject.

It has been obvious for a long time that on many interpretations of 'rational', both the scientist and the diviner act rationally, in their contexts. And it is likewise trivial to say that the diviner "thinks differently" from the scientist, much as it is trivial to say that the scientist "thinks differently" when preparing an egg than when preparing a petri dish. To make her claim nontrivial, the defender of the claim that the diviner and the scientist have alternative standards of rationality faces a difficult, if ultimately promising, task. A crucial part of the task, of course, is to identify significant and systematic differences between the diviner's culture and the relevant community of contemporary scientists. This is difficult enough, but perhaps the trickier and more amorphous part of the task is to show whether, when, and why such differences should count as pragmatic grounds for differences in their respective species of rationality. 


\section{References}

Ariely, Dan

2009 Predictably Irrational. New York: HarperCollins.

Barnes, Barry, and David Bloor

1982 Relativism, Rationalism, and the Sociology of Knowledge. In Rationality and Relativism, edited by M. Hollis and S. Lukes. Cambridge: MIT.

Bauer, Nancy 2005

On Human Understanding. In Wittgensteinian Fideism?, edited by K. Nielsen and D. Z. Phillips. London: SCM Press.

Beattie, John H. M.

1964

Other Cultures. New York: Free Press.

1966 Ritual and Social Change. Man: The Journal of the Royal Anthropological Institute 1:60-74.

1970 Reason and Ritual. In Rationality, edited by B. Wilson. Evanston: Harper \& Row.

Boghossian, Paul A. 2006

Fear of Knowledge. Oxford: Oxford University Press.

Boroditsky, Lera 2001

Does Language Shape Thought? English and Mandarin Speakers' Conceptions of Time. Cognitive Psychology 43 (1):1-22. 
Chang, Kwang-Chih

1980

Press.

Cooper, David

1975
Shang Civilization. Cambridge: Harvard University

Da Costa, Newton and French, Steven 1995

Partial Structures and the Logic of Azande. American Philosophical Quarterly 32 (4):325-339.

Davidson, Donald 1973

On the Very Idea of a Conceptual Scheme. Proceedings and Addresses of the American Philosophical Association 47:5-20.

Dretske, Fred

1981

Knowledge and the Flow of Information. 1st MIT Press ed. Cambridge, Mass.: MIT Press.

Elio, Renée

2002 Common Sense, Reasoning, and Rationality. Oxford: Oxford University Press.

Epstein, Brian

2010

History and the Critique of Social Concepts. Philosophy of the Social Sciences 40 (1): 3-29.

Evans-Pritchard, Edward Evan 
Zande Therapeutics. In Essays Presented to C.G. Seligman, edited by E. E. Evans-Pritchard, R. Firth, B. Malinowski and I. Schapera. London: Kegan Paul. Oxford: Clarendon Press.

Evans, Jonathan, and D. E. Over 1996 Rationality and Reasoning. Hove: Psychology Press.

Fadiman, Anne 1997 The Spirit Catches You and You Fall Down. New York: Farrar, Straus, and Giroux.

Firth, Raymond 1964 Essays on Social Organization and Values. London: Athlone Press.

Frankfurter, David 2005 Voices, Books, and Dreams: The Diversification Media in Late Antique Egypt. In Mantikê: Studies in Ancient Divination, edited by S. I. Johnston and P. T. Struck. Leiden: Brill.

Garcia-Carpintero, Manuel, and Max Kölbel, eds. 2008 Relative Truth. Oxford: Oxford University Press.

Goldman, Alvin 1980 The Internalist Conception of Justification. In Midwest Studies in Philosophy, edited by P. A. French, T. E. Uehling and H. K. Wettstein. 
1986 Epistemology and Cognition. Cambridge: Harvard University Press.

Goodman, Nelson 1978 Ways of Worldmaking. Indianapolis: Hackett.

Goody, J.

1961 Religion and Ritual: The Definitional Problem. American Journal of Sociology 12:142-164.

Graf, Fritz

2005 Rolling the Dice for an Answer. In Mantikê: Studies in Ancient Divination, edited by S. I. Johnston and P. T. Struck. Leiden: Brill.

Halivni, David

1991

Peshat and Derash: Plain and Applied Meaning in Rabbinic Exegesis. New York: Oxford University Press.

Harman, Gilbert 1995 Rationality. In Thinking: An Invitation to Cognitive Science, edited by E. E. Smith and D. N. Osherson. Cambridge: MIT Press.

2004 Practical Aspects of Theoretical Reasoning. In The Oxford Handbook of Rationality, edited by A. R. Mele and P. Rawling. Oxford: Oxford University Press.

Haslanger, Sally 2005

What are we Talking About? The Semantics and Politics of Social Kinds. Hypatia 20 (4):10-26. 
Hawley, Katherine, and Macpherson, Fiona 2010

The Admissible Contents of Experience. Oxford: Wiley Blackwell.

Hollis, Martin

1982

The Social Destruction of Reality. In Rationality and Relativism, edited by M. Hollis and S. Lukes. Cambridge: MIT Press.

Hollis, Martin, and Steven Lukes 1982

Rationality and Relativism. Cambridge: MIT Press.

Horton, Robin

1967

African Traditional Thought and Western Science. Africa 37 (1 and 2):50-71 and 155-187.

1993

Patterns of Thought in Africa and the West. Cambridge: Cambridge University Press.

Iles-Johnston, Sarah.

Delphi and the Dead. In Mantikê: Studies in Ancient Divination, edited by S. I. Johnston and P. T. Struck. Leiden: Brill.

Jarvie, I.C., and Agassi, Joseph 1970 [1967] The Problem of the Rationality of Magic. In Rationality. Evanston: Harper \& Row.

Johnson-Laird, Philip N. 
2004 Mental Models and Reasoning. In The Nature of Reasoning, edited by J. P. Leighton and R. J. Sternberg. Cambridge: Cambridge University Press.

Keightley, David N.

1988

Shang Divination and Metaphysics. Philosophy East and West 38 (4):367-397.

Kelly, Sean Dorrance

2007

What Do We See (When We Do)? In Reading Merleau-Ponty: On the Phenomenology of Perception, edited by T. Baldwin. Oxford: Routledge.

Kelly, Thomas

2003 Epistemic Rationality as Instrumental Rationality: A Critique. Philosophy and Phenomenological Research 66 (3):612-640.

Krausz, Michael and Meiland, Jack 1989 Relativism: Interpretation and Confrontation. Notre Dame: University of Notre Dame Press.

Krausz, Michael and Shusterman, Richard 1999 Interpretation, Relativism, and the Metaphysics of Culture: Themes in the Philosophy of Joseph Margolis. Amherst, NY: Humanity Books.

Kugel, James L. 2007 How to Read the Bible: A Guide to Scripture, Then and Now. New York: Free Press.

Kuhn, Thomas S. 
Kuhn, Thomas S., James Conant, and John Haugeland 2000 The Road Since Structure: Philosophical Essays, 1970-1993. Chicago: University of Chicago Press.

Kyburg, Henry

Leach, E.R. 1954 The Political Systems of Highland Burma. London: Bell.

Leighton, Jacqueline P., and Sternberg, Robert J. 2004 The Nature of Reasoning. Cambridge: Cambridge University Press.

Lucy, John 1996 Grammatical Categories and Cognition: A Case Study of the Linguistic Relativity Hypothesis. Cambridge: Cambridge University Press.

Lukes, Steven

1970 [1967] Some Problems about Rationality. In Rationality, edited by B. Wilson. Oxford: Blackwell.

1982 Relativism in its Place. In Rationality and Relativism, edited by M. Hollis and S. Lukes. Cambridge: MIT Press.

Manktelow, K. I., and D. E. Over 
Rationality: Psychological and Philosophical Perspectives. London: Routledge.

Meiland, Jack and Krausz, Michael 1982

Relativism: Cognitive and Moral. Notre Dame: University of Notre Dame Press.

Mele, Alfred R., and Piers Rawling 2004 The Oxford Handbook of Rationality. New York: Oxford University Press.

Millikan, Ruth Garrett 1984 Language, Thought, and Other Biological Categories: New Foundations for Realism. Cambridge: MIT Press.

Moscovitz, Leib 2002

Talmudic Reasoning: From Casuistics to Conceptualization. Tübingen: Mohr Siebeck.

Nielsen, Kai

1967 Wittgensteinian Fideism. Philosophy 42 (161):191209.

Nielsen, Kai, and D.Z. Phillips 2005 Wittgensteinian Fideism? London: SCM Press.

Peacocke, Christopher 1992 A Study of Concepts. Cambridge: MIT Press.

Peek, Philip, ed. 1991 African Divination Systems: Ways of Knowing. Bloomington: Indiana University Press. 
Plantinga, Alvin

$1993 \quad$ Warrant: The Current Debate. New York: Oxford University Press.

Preyer, Gerhard, and Georg Peter

2000 The Contextualization of Rationality: Problems, Concepts and Theories of Rationality. Paderborn: Mentis.

Pryor, James

2001

Highlights of Recent Epistemology. British Journal for the Philosophy of Science 52:95-124.

Putnam, Hilary

1975

The Meaning of 'Meaning'. In Philosophical Papers. Cambridge: Cambridge University Press.

1990 Realism with a Human Face. Cambridge, Mass.: Harvard University Press.

Recanati, Francois 2004

Pragmatics and Semantics. In Handbook of Pragmatics, edited by L. Horn and G. Ward. Oxford: Blackwell.

Rorty, Richard 1998 Davidson's Mental-Physical Distinction. In The Philosophy of Donald Davidson, edited by F. Hahn.

Rysiew, Patrick 
2008 Rationality Disputes - Psychology and Epistemology. Philosophy Compass 3 (6):1153-1176.

Samuels, Richard, Stephen P. Stich, and Michael Bishop

2002 Ending the Rationality Wars. In Common Sense, Reasoning, and Rationality, edited by R. Elio. Oxford: Oxford University Press.

Samuels, Richard, Stephen P. Stich, and Luc Faucher 2004 Reason and Rationality. In Handbook of Epistemology, edited by I. Niiniluoto, M. Sintonen and J. Wolenski. Dordrecht: Kluwer.

Schlesinger, I.M.

1991

The Wax and Wane of Whorfian Views. In The Influence of Language on Culture and Thought, edited by R. L. Cooper and B. Spolsky. New York: Mouton de Gruyter.

Schofer, Jonathan

2005 Exegesis, Divination, and the Foundations of Knowledge, ms.

Simon, Lawrence 1998 Rationality and Cultural Relativism. In Routledge Encyclopedia of Philosophy, edited by E. Craig. London: Routledge.

Smith, Jonathan Z. 1982

Imagining Religion: From Babylon to Jonestown. Chicago: University of Chicago Press. 
Smith, Michael

2004 Humean Rationality. In The Oxford Handbook of Rationality, edited by A. R. Mele and P. Rawling. Oxford: Oxford.

Smith, Richard J.

1991

Fortune-tellers and Philosophers: Divination in Traditional Chinese Society. Boulder: Westview Press.

Stein, Edward 1996 Without Good Reason: The Rationality Debate in Philosophy and Cognitive Science. Oxford: Clarendon Press.

Stern, Josef

2000

Metaphor in Context. Cambridge: MIT Press.

Stich, Stephen P.

The Fragmentation of Reason: Preface to a Pragmatic Theory of Cognitive Evaluation. Cambridge: MIT Press.

Struck, Peter T. 2005 Divination and Literary Criticism? In Mantikê: Studies in Ancient Divination, edited by S. I. Johnston and P. T. Struck. Leiden: Brill.

Tambiah, Stanley Jeyaraja

Magic, Science, Religion, and the Scope of Rationality. Cambridge: Cambridge University Press. 
Thaler, Richard, and Cass Sunstein

2008 Nudge. New Haven: Yale University Press.

Tso-pin, Tung, and Lien-sheng Yang

1948 Ten Examples of Early Tortoise-Shell Inscriptions.

Harvard Journal of Asiatic Studies 11 (1-2):119-129.

Tylor, Edward Burnett

1871 Primitive Culture. London: John Murray.

Ulin, Robert C.

2001 Understanding Cultures: Perspectives in Anthropology and Social Theory. 2nd ed. Malden: Blackwell.

von Neumann, John, and Oskar Morgenstern

1944 Theory of Games and Economic Behavior. Princeton: Princeton University Press.

Wilson, Bryan R.

1970 Rationality. Evanston: Harper \& Row. 